\title{
Dietrich Bonhoeffer como teólogo confesional
}

\author{
Manfred Svensson \\ UNIVERSIDAD DE LOS ANDES \\ msvensson@uandes.cl
}

Resumen: El presente artículo explora la relación de Bonhoeffer con los escritos confesionales luteranos, mostrando que no solo las Escrituras, sino también estos documentos del siglo XVI constituyen un foco de orientación fundamental a lo largo de su obra. Se discute asimismo su participación en la elaboración de la Confesión de Betel, el más temprano intento de este género por parte de la Iglesia Confesante en respuesta al régimen nacionalsocialista. Por último, se expone el afán (y las dificultades) de Bonhoeffer para dar con un término medio que lo aleje tanto de un confesionalismo rígido como de una relación no vinculante con las confesiones.

Palabras clave: Confesionalismo, Bonhoeffer, Confesión de Betel, Iglesia Confesante.

Abstract: The article discusses Bonhoeffer's relation to the Lutheran confessional writings. We show the degree to which not only Scripture, but also the Lutheran confessions, play a significant role in his orientation under the Nazi regime. We further discuss his role in the redaction of the Bethel Confession of Faith, the earliest of the confessional statements that emerged from the Confessing Church. Finally, we discuss Bonhoeffer's difficulties in his search for a middle ground that might keep him at a distance from a traditionalistic relation to the confessions while at the same time adhering to them in a binding way.

Keywords: Confessionalism, Bonhoeffer, Bethel Confession, Confessing Church. 


\section{INTRODUCCIÓN}

¿Qué clase de teólogo fue Dietrich Bonhoeffer? Tal pregunta puede ser leída en sentidos muy distintos. Puede entenderse como una pregunta respecto del carácter de su trabajo teológico, de modo que se responda, por ejemplo, que fue un "teólogo comprometido". Puede leerse también como pregunta respecto de escuelas teológicas, de modo que se responda atendiendo a las diversas tendencias que confluyen en su obra: se le puede describir entonces como un teólogo barthiano o semibarthiano. Como indica nuestro título, sugerimos aquí que Bonhoeffer puede ser leído como un teólogo confesional. ¿Responde dicha caracterización a alguna de estas dos maneras de abordar nuestra pregunta inicial? Aunque el adjetivo "confesional" no puede entenderse solo como referencia a un modo de trabajo teológico, su referencia a un documento confesionalmente vinculante desde luego incluye esa dimensión; tampoco puede entenderse como simple descripción de una escuela, pero una oposición entre teología confesional y teología liberal se suele presentar espontáneamente a la mente de quien oye el término. Como esperamos mostrar, tanto el modo en que Bonhoeffer realizó su trabajo como parte del fondo de su mensaje se ven iluminados si acudimos a esta categoría. Y aunque veremos que su confesionalidad es menos estricta de lo que el término sugiere, se trata de una dimensión relevante que no ha recibido la atención debida.

En efecto, este es un modo de entender su obra al que la literatura no recurre en absoluto. El hecho resulta llamativo, pues son numerosas las restantes alternativas que han circulado a la hora de clasificar a Bonhoeffer. Durante las primeras décadas tras su muerte no fue inusual tenerle por precursor de la teología de la liberación y de la muerte de Dios ${ }^{1}$. Tras eso vinieron alternativas conservadoras en la carrera por apropiarse de su legado. Georg Huntemann, por ejemplo, lo volvía un hombre de "law and order"2. Una variante algo más exitosa de ese proyecto -aunque con pretensiones no académicas- es la biografía de Eric Metaxas publicada el año 2011. En esta, Bonhoeffer es presentado como un hombre cuya vida entronca fácilmente con el mundo evangélico norteamericano. Acentuando

1 Para ilustraciones significativas véase G. GutiérRez, "Los límites de la teología moderna: un texto de Bonhoeffer", en Concilium, 145 (1979) 222-236 y J. A. T. Robinson, Honest to God (SCM Press, Londres, 1967).

2 G. Huntemann, Der Andere Bonhoeffer. Die Herausforderung des Modernismus (Brockhaus, Wuppertal, 1989) 207-210. 
una experiencia de conversión de Bonhoeffer en Harlem y ofreciendo más bien vagas referencias a su trasfondo teológico en Alemania, Metaxas presenta un Bonhoeffer considerablemente más conservador que el usual $^{3}$. Pero aquí no nos preocupan los variados aciertos y desaciertos de esta obra, sino los instrumentos con que cuenta Metaxas para presentar su comprensión de Bonhoeffer: lo vuelve "evangélico", pero no lo vuelve "confesional". En otras palabras, esta alternativa es ignorada por las interpretaciones evangélicas o conservadoras tanto como por las de tenor liberacionista. Todas esas interpretaciones tienen en común el ver en Bonhoeffer a alguien "confesante", pero caben dudas respecto de si ese término significa para ellas algo más que "militante".

Este hecho no debe sorprender. El problema no se encuentra en Bonhoeffer ni solo en la recepción de Bonhoeffer, sino en la pobre suerte que ha corrido la idea de iglesia o teología confesional para entender diversos fenómenos del protestantismo contemporáneo. No solo en la bibliografía específica sobre Bonhoeffer, sino también en las historias generales de la resistencia protestante contra el nacionalsocialismo esta es una dimensión que rara vez recibe la atención que merece ${ }^{4}$. El más equilibrado juicio al respecto es el ofrecido por Christoph Strohm. En sus palabras, "para el surgimiento de una resistencia de inspiración cristiana fue fundamental la relación complementaria entre el cristianismo de la religiosidad popular (volkskirchlich) y el de orientación confesional"5. Estas fuerzas de "orientación confesional" fueron fundamentales por su capacidad de resistencia tanto ante la violencia como ante la seducción ideológica, pero su capacidad de vínculo con grupos no confesionales fue lo determinante para el futuro. El peculiar confesionalismo de Bonhoeffer constituye un puente fundamental entre estos tipos de grupos. El presente artículo partirá, en consecuencia, con una explicación de lo que ha de entenderse bajo confesionalismo en la historia protestante. Se atenderá

3 Para una reseña crítica que pone el acento en este género de problema véase C. Green, "Hijacking Bonhoeffer" en The Christian Century, 4 de octubre de 2010. Para una valoración positiva véase J. BALLor, “The Many Bonhoeffers" en The City, 19 de septiembre de 2010.

4 Es elocuente la omisión de esta perspectiva en obras como la de V. BARNETT, For the Soul of the People. Protestant Protest Against Hitler (Oxford University Press, Oxford, 1998).

5 Сн. Sтrohm, Kirchen im Dritten Reich (C.H. Beck, München, 2011) 118. 
asimismo a las dificultades que ha habido para que se establezca como una herramienta de interpretación. En segundo lugar, se verá en qué sentidos resulta una categoría iluminadora para el estudio de Bonhoeffer. Esto es, se señalará en qué medida una tradición confesional se encuentra tras su obra, se considerará en qué medida el término entra en su propia autocomprensión, se analizará el papel desempeñado por Bonhoeffer en la redacción de la Confesión de Betel, y se atenderá a algunas controversias menores en que la perspectiva confesional también resulta relevante. Estas preguntas se plantean aquí primordialmente con miras a la comprensión de la obra del mismo Bonhoeffer. Contienen, sin embargo, también un interés contemporáneo para cada una de las tradiciones cristianas que se plantean el modo en que su específica confesionalidad se hace presente en el testimonio cristiano hoy.

\section{2. “Protestantismo Confesional” como Categoría de InTerpretación}

¿Qué papel desempeña la idea de un protestantismo confesional para comprender la naturaleza de las iglesias nacidas de la Reforma? Por lo pronto, puede notarse que este rótulo se encuentra más vigente en el trabajo historiográfico respecto de los nacientes estados modernos que en el trabajo puramente teológico. Durante las últimas cinco décadas ha sido, en efecto, corriente describir como "confesionalización" el proceso por el que se conformaron identidades nacionales distintivamente anglicanas, católico-romanas, luteranas o calvinistas ${ }^{6}$. En cambio, cuando se apela al carácter "confesional" de tal o cual tendencia teológica del protestantismo contemporáneo, tiende a ser en un espíritu de lamento o de ressourcement más que en un espíritu descriptivo ${ }^{7}$. Ahora bien, los procesos de confesionalización de los estados europeos y los procesos eclesiásticos internos de formación confesional desde luego fueron de la mano. Sin ir más lejos, hay una tendencia clara a que las fechas de consolidación de

6 Para el desarrollo de esta aproximación puede verse S. Dixon, Contesting the Reformation (Wiley-Blackwell, Oxford, 2012) 163-204.

7 Para un título inclinado al lamento véase I. Hamilton, The Erosion of Calvinist Orthodoxy: Drifting from the Truth in Confessional Scottish Churches (Mentor, Fearn, 2010). Para el proyecto de ressourcement véase S. CLARK, Recovering the Reformed Confessions (Presbyterian and Reformed Publishing, Phillipsburg, 2008). Para el caso luterano véase E. Gritsch y R. JENSON, Lutheranism: The Theological Movement and its Confessional Writings (Fortress Press, Philadelphia, 1975). 
los procesos confesionales coincidan con el cierre de procesos políticos de largo alcance. Si pensamos en el mundo inglés, por ejemplo, se puede considerar el lugar de la Asamblea de Westminster (1643-1652) en el contexto de la Guerra Civil Inglesa, o la coincidencia temporal de la Gloriosa Revolución y la Confesión Bautista de 1689. El vínculo entre perspectiva confesional y estado confesional es suficientemente fuerte como para que las crisis de una dimensión afectaran a la otra.

No se trata, sin embargo, de vínculos unidireccionales ${ }^{8}$. La crisis del protestantismo confesional no se inició por la crisis de la Europa de estados religiosamente homogéneos, crisis que después de todo es un fenómeno sumamente tardío. Bien puede decirse, por el contrario, que desde que existe el protestantismo, existe por una parte en una forma confesional y, por otra parte, en diversas combinaciones de erasmismo y reforma radical. Estas últimas acabarían por volverse predominantes, con frecuencia minando de modo silencioso las tradiciones confesionales. En cuestión se encuentran por supuesto comprensiones rivales de lo que podría significar el sola scriptura. Está claro que, para la tradición de las reformas magisteriales, dicha fórmula nunca estuvo en tensión con la elaboración de síntesis doctrinales robustas. Estas, por el contrario, son un fenómeno tan central como temprano, según puede constatarse por el amplio número de catecismos y confesiones de fe 9 . No en vano Jaroslav Pelikan ha escrito que es con la Reforma protestante que la "confesión de fe", como algo distinto de un credo, llega a establecerse como género literario y teológico ${ }^{10}$. Contra eso se levantaron, sin embargo, las tradiciones humanistas y radicales que en buena medida pueden ser caracterizadas por su crítica de las confesiones de fe, un punto que en sus respectivos programas guarda estrecha relación con el resto de su crítica a una política eclesiástica excesivamente formalizada. No cabe

8 K. Appold, por ejemplo, ha mostrado la frecuencia con que la vida universitaria, aunque fuertemente vinculada con la confesión de fe, se mueve en direcciones distintas de las previstas por los respectivos príncipes. Véase Appold, "Academic Life and Teaching in Post-Reformation Lutheranism” en R. KolB (ed.), Lutheran Ecclesiastical Culture 1550-1675 (Brill, Leiden, 2008) 65-116.

9 De 1522 a 1529 sesenta y dos impresiones de trece distintos textos de instrucción catequética son publicados en Wittenberg, según documenta T. WENGERT,

10 J. Pelikan, Credo: Historical and Theological Guide to Creeds and Confessions of Faith in the Christian Tradition (Yale University Press, New Haven y Londres, 2003) 458. 
aquí hacernos cargo de las múltiples causas y caras de ese fenómeno; pero sí cabe notar que al entrar en el siglo XVIII estas tendencias, hasta entonces reducidas a los márgenes del protestantismo, comenzaron a ser características de su centro. La creciente pérdida de importancia del protestantismo confesional, en tanto, llevó a que el mismo se volviera menos visible para la mirada retrospectiva, de modo que el conjunto del protestantismo comienza a ser visto como un factor en la irrupción de la modernidad más que como un tipo específico de confesionalidad. Bonhoeffer mismo escribiría contra ese género de interpretación de la Reforma en que "únicamente se acaba apreciando en el protestantismo la posibilidad de ser librepensadores" de espíritus huérfanos, refugio de la Ilustración inculta" ${ }^{12}$.

Ahora bien, para efectos del presente artículo resulta fundamental notar que, si bien hay un sentido en que el protestantismo confesional es un fenómeno de la modernidad temprana, que luego entró en el tipo de crisis que hemos reseñado, existe también lo que podemos llamar un "confesionalismo de segunda generación". Con tal término designamos a aquel confesionalismo que no solo continúa la tradición confesional precedente, sino que surge como respuesta consciente al triunfo de las vertientes tanto pietistas como liberales. El siglo XIX ha sido descrito por algunos como un siglo de secularización, y por otros como un siglo de grandes avivamientos religiosos independientes ${ }^{13}$. Pero el confesionalismo debe ser también incluido entre sus grandes tendencias, $\mathrm{y}$ de un modo que se hizo presente en todas las grandes confesiones ${ }^{14}$.

11 DBW IX, 109. Las obras de Bonhoeffer las citamos según la edición crítica alemana Dietrich Bonhoeffer Werke, 17 vols., ed. por E. BETHGE et al. Chr. Kaiser-Gütersloher Verlagshaus, München y Gütersloh, 1986-1999] por volumen y página (p. ej. DBW $\mathrm{V}, 34)$.

12 DBW IX, 109.

13 Para estas lecturas véase O. CHADwICK, The Secularization of the European Mind in the Nineteenth Century (Cambridge University Press, Cambridge, 1990) y M. Nolu, The Rise of Evangelicalism. The Age of Edwards, Whitefield, and the Wesleys (IVP Academic, Downers Grove, 2005).

14 Para la idea de una segunda era confesional véase O. BLASCHKE, "Das 19. Jahrhundert: Ein Zweites Konfessionelles Zeitalter?" Geschichte und Gegenwart, 26 (2000) 38-75. La más completa visión global del confesionalismo protestante del periodo sigue siendo W. H. Conser, Church and Confession. Conservative Theologians in Germany, England, and America 1815-1866 (Mercer University Press, Macon, 1984). 
Con diversos acentos según región y confesión, se trata de un fenómeno que se puede encontrar desde Alemania a los Estados Unidos ${ }^{15}$. Dicho confesionalismo podía ser crítico no solo de la naciente secularización o del liberalismo. También podía serlo respecto del individualismo que diagnosticaba en parte del avivamiento evangélico. Resulta además crucial notar que estos movimientos confesionales pueden encontrarse en variada relación con el mundo moderno en lo que a política y cultura se refiere. La caracterización de sus miembros como "conservadores" se encuentra justificada en algunos sentidos, pero si se usa dicho epíteto como caracterización principal se oscurece el carácter preeminente de su autocomprensión como movimientos confesionales, movimientos que pueden no ser conservadores en otros sentidos. Así, mientras algunos representantes del renacimiento confesional fueron críticos radicales de las revoluciones democráticas, otros fueron promotores de partidos de masas y defensores del pluralismo ${ }^{16}$.

Este último es, por ejemplo, el caso del neocalvinismo holandés. Abraham Kuyper (1837-1920), su principal articulador, se caracteriza por un programa que bien puede ser considerado como un confesionalismo de segunda generación, un confesionalismo con fuerte conciencia de su inserción en una sociedad plural, con una reflexión política que busca defender la pluralidad de esferas sociales precisamente como vía de articulación de confesiones rivales en un contexto moderno ${ }^{17}$. En este caso se trata además de una articulación moderna del confesionalismo que perdura hasta hoy ${ }^{18}$. A lo largo y ancho de Europa, en efecto, los movimientos confesionales mantuvieron vínculos y se observaron recíprocamente. Las líneas de continuidad en el paso al siglo XX no siempre

15 Para el escenario norteamericano véase D. G. HART, The Lost Soul of American Protestantism (Rowman \& Littlefield, Lanham, 2002).

16 Para estos contrastes véase Conser, Church and Confession.

17 Para introducción véase J. BRATT, Abraham Kuyper: Christian Democrat, Modern Calvinist (Eerdmans, Grand Rapids, 2013).

18 Para una ilustración contemporánea de cómo se trata ahí el pluralismo véase J. Chaplin, 'Rejecting Neutrality, Respecting Diversity. From "Liberal Pluralism" to Christian Pluralism', Christian Scholar's Review, 35 (2006) 143-76. 
fueron fuertes, pero sí había conciencia de ellas ${ }^{19}$. Significativamente, a Bonhoeffer esta conexión le es planteada a modo de advertencia. En efecto, Helmut Rößler, un anterior compañero de estudios suyo, evoca el caso holandés en tono de marcada preocupación: "lo que ocurre hoy en Alemania ya tuvo una versión hace exactamente cien años en Holanda, en la escisión de los 'Gereformeerden' [el nombre del movimiento de Kuyper]" ${ }^{\prime 20}$. El parecer de Rößler, expresado en carta de 1934 a Bonhoeffer, es que este neocalvinismo holandés debía servir no de inspiración, sino de advertencia por la pérdida de influencia sobre la vida del pueblo que la Iglesia así habría sufrido en los Países Bajos ${ }^{21}$. Se trata de una advertencia elocuente respecto de la tensión que se desarrollaría entre la iglesia confesante y el movimiento nacionalsocialista.

\section{Contexto político y temprana relación de Bonhoeffer CON EL Confesionalismo}

Tras esta breve reseña del auge, caída y restauraciones de la teología confesional, podemos volver la mirada a Bonhoeffer. No es el candidato más plausible para ser presentado como ícono de la teología confesional, y esto por varias razones. En primer lugar, cabe notar que la teología confesional es un producto del esfuerzo de las grandes confesiones por delimitarse recíprocamente. Bonhoeffer se encuentra, en contraste, involucrado de diversos modos en proyectos de tenor opuesto. Participa del naciente movimiento ecuménico y, como fruto de la oposición al nacionalsocialismo, es parte de la Iglesia Confesante (Bekennende Kirche) que incorporó en su seno tanto a luteranos como calvinistas. Además,

19 Para un estudio circunscrito al caso alemán véase H.-J. REESE, Bekenntnis und Bekennen. Vom 19. Jabrbundert zum Kirchenkampf der nationalsozialistischen Zeit (Vandenhoeck \& Ruprecht, Gotinga, 1974).

20 DBW XIII, 255. El término holandés designa a la tradición confesional que se escinde de la iglesia nacional holandesa en 1892.

21 Paralelos entre neocalvinismo holandés y Bonhoeffer han sido recientemente objeto de más atención. Véase G. Dekker y G. Harinck. "The Position of the Church as Institute in Society: A Comparison between Bonhoeffer and Kuyper" en The Princeton Seminary Bulletin 28, 1 (2007) 86-98. Asimismo J. A. GarCia, "The Pulpit, the Lectern, and the Sickbed. Comparing Dietrich Bonhoeffer and Hermann Bavinck on Church and Academy" en The Kuyper Center Review. Volume Five: Church and Academy (Eerdmans, Grands Rapids, 2015) 44-61. 
incluso si el espíritu de su obra fuese afín a un proyecto distintivamente confesional, en la forma parece no serlo. En efecto, no parece equivocado decir que, por razones estrechamente relacionadas con la naturaleza del proyecto en cuestión, la teología confesional tiene en la teología sistemática su género característico, tanto como la tradición erasmista privilegia otros géneros y, particularmente, una oposición entre teología sistemática y teología bíblica ${ }^{22}$. Bonhoeffer, como fácilmente se puede constatar, cultiva una serie de géneros teológicos, pero no estaba en su horizonte la redacción de una Summa. Tanto por inclinación eclesiástica como por talante intelectual, la sugerencia del presente artículo parecería, pues, descaminada. Estas dos objeciones son válidas. Su validez, con todo, es parcial. En primer lugar, porque la preocupación ecuménica no es en lo más mínimo ajena a la tradición confesional previa; en segundo lugar, porque si bien Bonhoeffer no escribe una teología sistemática, sí está involucrado en la redacción de una confesión de $\mathrm{fe}^{23}$. Antes de atender a ésta, consideraremos algo del trasfondo político y biográfico en que su obra empieza a articularse.

Hans-Jörg Reese ha llamado la atención sobre el hecho de que el "movimiento de fe de los germano-cristianos" (Deutsche Christen) -el movimiento eclesiástico que encarnaría la cosmovisión nacionalsocialista en los conflictos intraeclesiásticos-, cuenta con una visión de mundo, pero no cuenta con un credo explícito. Más bien es recurrente encontrarse en sus textos con fórmulas según las cuales la "confesión de fe" debe ser reemplazada por una "confesión de vida" 24 . Parte de la política religiosa del régimen nacionalsocialista, en efecto, se articuló en torno al concepto

22 Para el temprano desarrollo de estas tradiciones rivales sigue siendo instructivo $\mathrm{H}$. Graf ReventLow, The Authority of the Bible and the Rise of the Modern World (SCM Press, Londres, 1984).

23. La dimensión ecuménica del confesionalismo del siglo XIX, que surge de un común descubrimiento del pasado perdido, ha sido tocada (contra la exclusiva identificación entre movimiento romántico y catolicismo romano) por F. W. KANTZENBACH, "Protestantische Geisteskultur und Konfessionalismus im 19. Jahrhundert" en A. Rauscher (ed.), Probleme des Konfessionalismus in Deutschland seit 1800 (Schöningh, Paderborn, 1984) 9-29, sobre todo 9-13.

24 H. J. ReEse, "Bekenntnis und Bekennen im Kirchenkampf" en M. Brecht y R. SCHWARz (eds.), Bekenntnis und Einheit der Kirche. Studien zum Konkordienbuch (Calwer Verlag, Stuttgart, 1980) 467-490. 470. 
de "desconfesionalización" (Entkonfessionalisierung). El hecho de que la retórica nacionalsocialista no fuera siempre abiertamente anticristiana es compatible con esta desconfesionalización, pues la propuesta era precisamente la de un "cristianismo positivo", nutrido de una religiosidad popular que a la vez podía ser fuertemente antieclesial. Este punto había incluso llegado a quedar consignado en el programa del partido nacionalsocialista, que en 1920 manifestaba su adhesión a este cristianismo positivo sin vínculo confesional alguno ${ }^{25}$. Ya el hecho de presentarse como la legítima iglesia protestante en Alemania implicó por parte de la Iglesia Confesante una ruptura con ese tipo de religiosidad.

En efecto, este tipo de escenario explica la intensidad con que una parte de los cristianos opositores al nacionalsocialismo se vuelca sobre las confesiones de fe. Con Jaroslav Pelikan cabe además notar que esta explosión de declaraciones confesionales bajo el Tercer Reich no tiene contraparte en otras persecuciones vividas por el cristianismo en el siglo XX. Hubo confesores y actos de confesión bajo persecuciones comunistas, pero curiosamente nada de confesiones formales de fe como las redactadas bajo el nacionalsocialismo ${ }^{26}$.

Junto a estas vicisitudes políticas, había elementos en la biografía de Bonhoeffer que lo ponían en contacto con la tradición confesional. Su bisabuelo Karl August von Hase fue la figura más imponente de la facultad de teología en Jena en el siglo XIX. Von Hase no podría en sentido alguno ser clasificado como un teólogo confesional, estando más bien marcado por Schleiermacher y Herder. Con todo, potenciado así por el romanticismo a una preocupación por la historia del cristianismo, se deben a él algunas de las más significativas recopilaciones de los escritos confesionales y de la escolástica luterana publicadas en el siglo XIX ${ }^{27}$. En él Bonhoeffer podía tener no la imagen de un teólogo confesional, pero sí la idea de que también para los teólogos modernos el ocuparse de este fenómeno podía ser una tarea digna de emprender.

Parte de su temprana experiencia como estudiante puede haberlo

25 Strohm, Die Kirchen im Dritten Reich 12-15.

26 Pelikan, Credo 223.

27 Su principal texto a este respecto es Hutterus Redivivus oder Dogmatik der evangelischlutherischen Kirche (Sühring, Leipzig, 1829). 
movido en la misma dirección. Tras su estadía de intercambio en Estados Unidos, durante 1931, presentó en Alemania un informe que ha adquirido cierta celebridad por la manera incisiva en que analiza el contexto teológico y filosófico norteamericano. Entre los diversos elementos a los que Bonhoeffer atiende se encuentra precisamente el carácter no confesional del protestantismo americano. Cabe aquí recordar que, tras su segunda estadía en Estados Unidos, ocho años más tarde, Bonhoeffer escribiría un segundo balance, esta vez bajo el título "Protestantismo sin Reforma"28. Bien puede decirse que este modo de describir dicho protestantismo se encuentra ya presente en el informe de su primera estadía, donde habla de un protestantismo casi no tocado por la Reforma. Bonhoeffer fundamenta este juicio sobre todo en la ausencia de credos explícitos. Por decirlo de otro modo, para Bonhoeffer el haber sido tocado por la Reforma significa ser confesional. Las denominaciones norteamericanas, en cambio, las describe como caracterizadas por ritos o posiciones sociales, pero "no por credo, dogma, ni sistema dogmático" 29 .

Bonhoeffer no se queda en esta constatación del espíritu antidogmático del cristianismo norteamericano, sino que lo vincula de modo expreso al "universal individualismo religioso" que se encontraría en estas tradiciones disidentes $^{30}$. "El Espíritu de Dios es entendido de un modo que no lo vincula decisivamente a la Palabra, por lo que no entienden lo que son un sermón, una confesión, un dogma, una Iglesia, la comunión"’31. Así puede verse que esta temprana posición confesional de Bonhoeffer frente a una alternativa antidogmática entronca de modo decisivo con el carácter eclesial de la fe cristiana. Es de la afirmación de este carácter, no de un dogmatismo abstracto, que se sigue la perspectiva confesional. Así, no es de extrañar que este confesionalismo se oponga de modo

\footnotetext{
28 DBW XV, 431-460.

29 DBW X, 276.

30 DBW X, 276.

31 DBW X, 276.
} 
enfático al fundamentalismo con el que en ocasiones se le confunde ${ }^{32}$. Cuando Bonhoeffer escribe sobre el movimiento fundamentalista no subraya en modo alguno el "conservadurismo" del mismo -que podría emparentarlo con el confesionalismo-, sino el individualismo que une a fundamentalistas con liberales ${ }^{33}$. En el reporte que escribiría tras su siguiente visita a Estados Unidos, en 1939, vuelve sobre estos rasgos de la religiosidad del país y nota que, si bien hay ahí simpatía por quienes, como él, se encuentran en una condición de oposición o minoría, "no hay comprensión alguna por una lucha confesional" ${ }^{34}$. Como bien señala Bonhoeffer a continuación, ésa es una perspectiva muy insatisfactoria para la víctima que no está tan preocupada de su propio destino como de la verdad de su causa. Dirijámonos, pues, al modo en que Bonhoeffer se ve involucrado en la "lucha confesional" en su propia Alemania.

\section{La Confesión de Betel}

A la luz de lo anterior, no es de extrañar que la respuesta de Bonhoeffer al régimen nacionalsocialista $-\mathrm{y}$ al partido "germano-cristiano" en la política eclesiástica- se sustentara desde temprano en una base confesional. Uno de los puntos más conocidos de esta oposición confesional al nacionalsocialismo es la redacción de la Confesión de Barmen, cuyo redactor principal fue Karl Barth. Pero aquí nos interesa dirigir la mirada a la Confesión de Betel, del año anterior, cuyo principal impulsor fue Bonhoeffer. La Confesión de Barmen usualmente ha opacado la de Betel. Pero de ésta cabe destacar, en primer lugar, que en su forma responde de modo más estricto a lo que suelen ser los documentos confesionales,

32 La confusión es denunciada también por confesionalistas norteamericanos que eran confundidos con el fundamentalismo en medio de la querella liberal-fundamentalista de comienzos de siglo. El caso más significativo para comparación con Bonhoeffer es el de J Gresham Machen, cuya descripción del protestantismo norteamericano, contenida en su obra Cristianismo y liberalismo, se presta para múltiples comparaciones con el análisis de Bonhoeffer tras sus estadías en dicho país. Tal como en el caso de Bonhoeffer, se trata de un confesionalismo que puede ir de la mano de una significativa aceptación de la modernidad cultural. Para una adecuada exposición véase D. G. HART, "When is a Fundamentalist a Modernist? J. Gresham Machen, Cultural Modernism, and Conservative Protestantism" en Journal of the American Academy of Religion 65, 4 (1997) 605-633.

33 DBW X, 277.

34 DBW XV, 443. 
al provenir de un conjunto de plumas en lugar de expresar el programa de un teólogo específico como Barth (un hecho, en efecto, que impidió a muchos luteranos unirse a la de Barmen). En segundo lugar, es digno de nota que fuera la Confesión de Betel, y no la de Barmen, la que estuviera dispuesta a hacer mención específica de los padecimientos de los judíos bajo el régimen nacionalsocialista (y esto en el primer año del mismo), un punto en el que Bonhoeffer ciertamente vio con más lucidez que Barth ${ }^{35}$. El proceso de redacción de esta confesión, y la relación de Bonhoeffer con sus otros autores (Georg Merz, Hermann Sasse y Wilhelm Vischer), vierte por lo mismo especial luz sobre el posicionamiento confesional de su teología.

A comienzos de agosto de 1933 un grupo de estudiantes de teología de Berlín escribe a Friedrich von Bodelschwing, director de los centros asistenciales de Betel (el principal centro luterano de asistencia a personas con discapacidad), solicitándole que, dada su autoridad, asumiera la dirección de lo que los alumnos consideraban una necesidad ineludible en medio del Kirchenkampf: la redacción de una nueva confesión, "que siente bases y tome posición respecto de los problemas de la Iglesia de hoy"36. Los estudiantes estaban claramente hablando de una confesión producida por un partido de la Iglesia, no emanada de los órganos centrales de la misma, pues lo que esperaban de tal confesión era precisamente forzar a esos órganos a una reacción ante el documento y así lograr una clarificación. Se trata, como señalamos, de un proyecto distintivamente luterano y que pretendía mantenerse como tal: los estudiantes piden a Bodelschwing que incluya en su redacción a Sasse y a Bonhoeffer - por quien afirman aquí haber sido profundamente influenciados-, y manifiestan también su deseo de que "nuestro mayor teólogo, Karl Barth, haga algo paralelo para la iglesia reformada" ${ }^{37}$. Cabe, con todo, notar que al ser escrita esta carta, Bonhoeffer ya sabía que se le esperaba para trabajar en Betel, por lo que no es posible determinar hoy cómo y por quién fue invitado.

35 Para este punto véase J. BALLOR, "The Aryan clause, the Confessing Church, and the ecumenical movement: Barth and Bonhoeffer on natural theology, 1933-1935" en Scottish Journal of Theology 59, 3 (2006) 263-280.

36 DBW XII, 109.

37 DBW XII, 109-110. Mi cursiva. "Reformada" significa aquí -como en todas estas fuentes- "calvinista". 
Del proceso de redacción de la Confesión de Betel se conservan cinco textos: desde un borrador preliminar y una primera versión, cuya redacción corresponde de modo primordial a Sasse y Bonhoeffer, hasta la versión definitiva, que ninguno de ellos estuvo dispuesto a firmar. Si el impulso original había sido el de poner a los germano-cristianos ante una confesión explícita, que los obligara a una toma de posición, Von Bodelschwing acabaría encaminando el proceso en una dirección algo distinta, intentando que la confesión fuese ante todo una ocasión para unificar posiciones entre los distintos partidos en que estaba dividido el luteranismo. Por lo mismo, se acabó incorporando las observaciones de una veintena de otros teólogos, de un modo que debilitó severamente los párrafos explícitamente dedicados a la cuestión judía. En lugar de "poner a los 'germano-cristianos' ante la pregunta confesional", como rezaba la intención de los primeros impulsores de la iniciativa, en lugar de presentar la confesión como documento vinculante, se acabó presentándola como ayuda para la reflexión ${ }^{38}$. La decepción de Bonhoeffer por este hecho fue uno de los principales factores tras su pronta partida a Inglaterra.

La participación de Bonhoeffer en la redacción de este documento nos permite evaluar su relación con un proceso de declaración confesional tanto por la naturaleza del texto mismo como por la relación que tuvo con los otros actores involucrados. Si dirigimos, en primer lugar, la mirada al texto, cabe notar que en su forma es considerablemente más cercano que la Confesión de Barmen a una clásica confesión de fe. Bien cabría decir que, en comparación con Barmen, la Confesión de Betel es por una parte más general y por otra más específica. Su especificidad se manifiesta, naturalmente, en la decisión con que el texto habla respecto de la relación con el pueblo judío ${ }^{39}$, y en su rechazo a entender la lucha como la ley fundamental de la existencia ${ }^{40}$. También la Confesión de Barmen contiene un desafío contra la pretensión de soberanía absoluta del régimen nacionalsocialista en su célebre artículo primero contra el reconocimiento "de otros poderes y eventos, figuras y verdades", distintos de la Palabra de Dios, como fuente

38 Para el desarrollo de la redacción, el papel desempeñado por los distintos actores, y sinopsis de las cinco variantes que se ha conservado del texto, véase CH.-R. MülLER, Bekenntnis und Bekennen. Dietrich Bonhoeffer in Bethel (1933). Ein lutherischer Versuch (Chr. Kaiser Verlag, München, 1989) y la síntesis de este trabajo en DBW XII, 503-507.

39 DBW XII, 402-406.

40 DBW XII, 373. 
de revelación. Pero carece de una mención igualmente clara de la situación de los judíos. Sin embargo, su discusión de la cuestión judía no convierte al texto de Betel en uno puramente circunstancial. Resulta más bien llamativa la necesidad que sus autores percibieron de insertar su reclamo por la situación de los judíos en una exposición general de la fe cristiana. Como confesión sigue en términos generales la estructura de los otros textos del género (Sagradas Escrituras, creación y caída, la redención y la Iglesia), y se apoya también de modo recurrente en los textos confesionales luteranos del siglo XVI. Ese tipo de tratamiento conjunto de lo permanente y de lo urgente puede tener efectos correctivos que fácilmente pasamos hoy por alto. El lenguaje seudoreligioso de una "providencia" que orienta el actuar del pueblo, frecuente entre los "germano-cristianos", es por ejemplo más fácilmente contrarrestado por la presentación de la doctrina de la providencia en un marco compartido con otros tópicos teológicos.

La existencia de documentos confesionales es ella misma objeto de reflexión en este texto. Resulta significativo, de hecho, el lugar en el que es tratado dicho tópico. Lo común, cuando el tópico es tematizado en confesiones protestantes, es una temprana discusión en el marco de la discusión sobre las Escrituras como principio formal de la Reforma. En el caso de la Confesión de Betel, en cambio, la única mención se encuentra en el extenso capítulo final sobre el Espíritu Santo y la Iglesia (VI), en concreto en la discusión sobre el ministerio ordenado (VI, 3.b). El papel que desempeñan las confesiones ahí es precisamente el de contribuir a dejar clara la subordinación de la autoridad pastoral a las Escrituras, y prevenir el entusiasmo ante el modelo contemporáneo de liderazgo (Führungsprinzip) en cualquiera de sus formas. El oficio de los ministros es de servicio a la Palabra de reconciliación, y no reciben su tarea de la nación ni de movimiento político o espiritual alguno. "Está sujeto a la ley de las Escrituras y de las confesiones, y no puede por tanto ordenar que se desaten los vínculos que unen la comunidad a la confesión" ${ }^{41}$. Queda aquí claro que el carácter confesional no es nada accidental al proyecto que se promueve, y tampoco es mera herencia acríticamente recibida del protestantismo temprano.

$41 \quad$ DBW XII, 398. 
La posición de Bonhoeffer también puede ser analizada a partir de su relación con los restantes redactores, en particular con Hermann Sasse, que de los implicados es quien más claramente sigue una trayectoria confesionalista en su carrera posterior ${ }^{42}$. La relación entre ambos resulta, en efecto, de importancia cardinal para iluminar la posición teológica de Bonhoeffer. Ambos crecieron en la iglesia de la unión prusiana, la forzada unificación estatal de calvinistas y luteranos que desde comienzos del siglo XIX constituyó un caso paradigmático de cristianismo no confesional. Al llegar a su madurez, en cambio, ambos reaccionaron contra dicho contexto, Bonhoeffer mediante el influjo barthiano y Sasse adhiriendo al renacimiento confesional luterano. Cuánta tensión se considere que hay entre dichas dos posiciones depende de cómo se interprete a Barth, el sentido de cuya obra resulta bastante menos transparente que la de Sasse. Su obra ha sido leída como una recuperaración del antiguo confesionalismo protestante, pero también como una revisión tal del mismo, que la fidelidad a la tradición es reemplazada por la sola revisión o el diálogo con la misma ${ }^{43}$.

Determinar el alcance preciso de la posición de Barth no es nuestro objeto aquí. El punto es simplemente comprender la tensión respecto de un confesionalismo más estricto como el de Sasse, y el singular lugar en que así Bonhoeffer queda entre Barth y éste. La relación entre Bonhoeffer y Sasse permitió su trabajo en común, como se dio en la primera etapa de la redacción de la Confesión de Betel, aunque no se tratara de alguien a quien Bonhoeffer tratara como autoridad (como sí siguió ocurriendo con Barth). Tras la Confesión de Betel se mantendrían en una relación que puede caracterizarse por cierta oposición, pero una que pasaba por atenta lectura y consideración recíproca. Lo que más nos importa aquí es que dicha tensión guarda relación precisamente con aquello que aquí tratamos: la percepción, de parte de Bonhoeffer, de que el confesionalismo de Sasse pecaba por excesiva rigidez y la percepción, de parte de Sasse, de que las alteradas circunstancias históricas se volverían en el caso de Bonhoeffer

42 Para su relación recíproca D. J. Webber, "Bonhoeffer and Sasse as Confessors and Churchmen", Logia 21, 4 (2012) 13-20.

43 Para esta última interpretación véase R. Glomsrud, "Karl Barth and Modern Protestantism: The Radical Impulse” en Scott Clark y Joel Kim, Always Reformed. Essays in Honor of W. Robert Godfrey (Westminster Seminary California, Escondido, 2010) 92-115. 
suficientes para transformar las diferencias confesionales en meras diferencias de escuela. La objeción de Sasse es, pues, por la medida en que la oposición al nacionalsocialismo había alterado la mirada de Bonhoeffer respecto de las relaciones entre luteranos y calvinistas. No cabe minimizar este tipo de oposición entre ambos. Pero sí cabe notar la importancia concedida por ambos a la confesión como criterio de posicionamiento. En medio de la usual crítica de Bonhoeffer a Sasse, siempre encontraba en su obra algo que considerara singularmente valioso. Aunque el confesionalismo de Sasse era más estricto que el de Bonhoeffer, el hecho de descubrir que no se trataba de un conservadurismo irreflexivo es lo que parece haber vuelto a Bonhoeffer un siempre atento lector de su colega $^{44}$.

\section{Herejía, Status Confessionis, y Uso de los Documentos Confesionales}

Al comenzar los años 30, Bonhoeffer ya se ha relacionado críticamente con cada una de las variantes no confesionales del protestantismo: en primer lugar, por cuna y estudio, ha conocido el cristianismo no confesional en su versión académica y liberal; en segundo lugar, su período en Estados Unidos lo ha confrontado con sus variantes evangélicas, fundamentalistas e individualistas; al volver a Alemania, se lo ha cruzado en el discurso populista de los germano-cristianos, en el programa de desconfesionalización o confesión vital en lugar de confesión de fe. La Confesión de Betel está escrita de un modo que marca distancia respecto de cada una de estas posiciones. Con este trasfondo en mente resultará, en efecto, fácil comprender la frecuencia y franqueza con que durante los años que siguen Bonhoeffer escribe citando los escritos confesionales y presionando por un trato respecto de los mismos como documentos vinculantes.

En septiembre de 1933 Bonhoeffer fue uno de los firmantes de la protesta contra la introducción de la cláusula aria -la sección de la Ley de Servicio Civil que proscribía a los judíos del servicio público- en los

44 La relación entre ambos se encuentra adecuadamente documentada a lo largo de la clásica biografía de Bethge. E Bethge, Dietrich Bonhoeffer. Theologe, Christ, Zeitgenosse (Chr. Kaiser Verlag, München, 1967). 
órganos eclesiásticos. El texto de dicha protesta termina con la singular afirmación de que "no vamos a cesar de levantar la voz contra cada vulneración de la confesión" ${ }^{45}$. Que la objeción contra la cláusula aria sea formulada en tales términos puede resultar difícil de comprender, pero no se trata de una excepción. Se trata en realidad de una frase tomada de modo casi literal de la red de emergencia de pastores (Pfarrernotbund) creada ese mismo mes -de la que emergería de hecho la Iglesia Confesante-, por la que los pastores se comprometían a "protestar sin reserva contra toda vulneración de la confesión" ${ }^{46}$. Quien tenga en mente este hecho, podrá comprender con mayor facilidad el hecho de que la batalla de Bonhoeffer contra los germano-cristianos se diera en una medida significativa imputándoles un carácter herético. La crítica era en realidad recíproca, y poseemos notas de respuesta de Bonhoeffer a la pregunta " ¿Herejía en la Iglesia Confesante?" Pero la crítica apenas podía tener sentido cuando era proferida por un movimiento expresamente anticonfesional. En efecto, Bonhoeffer describe dicho intento de sus adversarios como un esfuerzo por "golpear a la Iglesia Confesante con sus propias armas" ${ }^{47}$.

No cabe aquí hacernos cargo de modo exhaustivo de la apelación de Bonhoeffer a los textos confesionales, pero sí puede resultar iluminador detenernos en momentos que nos permitan estudiar su relación con éstos tanto al momento de iniciarse las tensiones con el nacionalsocialismo como cuando ya se ha iniciado la guerra. Teniendo presentes textos de 1933, 1940 y 1943, consideraremos, pues, primero la idea de un status confessionis, en segundo lugar, un enfrentamiento puntual en que Bonhoeffer es criticado por su uso de las confesiones luteranas y, finalmente, el lugar que ve para las mismas tras la guerra. El término status confessionis debe, en buena medida, su existencia en el actual discurso teológico protestante a Bonhoeffer. No se trata, con todo, de una completa novedad suya, sino de un concepto acuñado en la temprana historia del luteranismo. Su contexto de origen es el de los Interim de Ausburgo y Leipzig, normas imperiales transitorias por las que Carlos V procuró mantener la unidad religiosa del imperio tras la derrota militar luterana en 1547. Según el parecer de algunos teólogos

\footnotetext{
45 DBW XII, 144.

46 DBW XII, 144, n. 9.

47 DBW XIV, 701.
} 
luteranos del momento, dichas normas eran aceptables al solo requerir de ellos concesiones en asuntos indiferentes; otros, como Matthias Flacius, sostuvieron que en una "situación de confesión" los asuntos indiferentes dejan de serlo. La posición de Flacius es de hecho recogida en la Fórmula de Concordia de 1577, vía por la que la conoce Bonhoeffer (ahí expresado como casus confessionis $)^{48}$. ¿Pero qué es un status confessionis? Según sugiere la fórmula de Flacius de la que arranca la discusión, parece ser un término que designa la alteración, a la luz de circunstancias extraordinarias, del normal juicio de la Iglesia. Que las circunstancias son un factor relevante a considerar al determinar la moralidad de un acto -como en general ha sido enseñado por la tradición teológico-moral ${ }^{49}$ - parece así ser el general marco de referencia. Pero son circunstancias radicales las que aquí parecen ser tenidas en consideración. Ahora bien, ¿puede cualquier desafío a la propia confesión ser entendido como configurador de tal status, o se trata más bien de la identificación de cierto kairos único por el que se percibe estar en "la hora de la verdad"?. Ambas alternativas presentan riesgos que no podemos discutir aquí. En el primer caso, tenemos el riesgo de que cada conflicto posible elimine las zonas grises de lo indiferente. En el segundo caso, está el riesgo, no menor, de que la propia bybris sea la que lleve a apresuradamente identificar un status confessionis donde no lo hay. No es fácil ver cómo están articulados estos aspectos en Bonhoeffer, y no es extraño que alguien como Sasse viera aquí un riesgo de minimización de la doctrina (el cambio de status haría, recordemos, que lo que en un momento es diferencia entre iglesias, en otro se volviera diferencia entre escuelas $)^{50}$.

En cualquier caso, las primeras reflexiones de Bonhoeffer al respecto, en el año 1933, apuntan inequívocamente a usar la fórmula de un modo que traiga claridad. La fórmula aparece -aunque sin ser objeto de una discusión detenida- en el ensayo de junio sobre "La Iglesia ante la cuestión judía" ${ }^{51}$. Un memorándum de julio del mismo año, escrito por Bonhoeffer en inglés para informar a sus pares en el extranjero, usa la expresión en el

48 La fórmula según la cual nibil est adiaphoron in casu confessionis et scandali se encuentra recogida en Libro de Concordia, solida declaratio, $\mathrm{x}$.

49 Véase, por ejemplo, Tomás de Aquino, Summa theologiae I-II, q.18, a. 9 y 10.

50 Para la extensa discusión de este punto véase el ensayo de Bonhoeffer "Zur Frage der Kirchengemeinschaft" en DBW XIV, 655-691.

51 DBW XII, 354. 
título: "La cuestión judeo-cristiana como status confessionis". Ahí el sentido de la fórmula es ante todo el de contribuir a la claridad en el discernimiento a quien está siendo engañado con consideraciones sobre la importancia relativa del punto específico en discusión. Bonhoeffer advierte ahí que la sustancia misma de la Iglesia se jugaría con frecuencia en materias que parecen poco importantes ${ }^{52}$. Es solo captando "la sustancia espiritual" de la introducción del parágrafo ario que se estaría en condiciones de dar la "respuesta espiritual" adecuada ${ }^{53}$. En septiembre de 1933, ante la efectiva introducción del parágrafo ario, Bonhoeffer escribe a Barth afirmando que tal status ya está dado; Barth ve también el status confessionis como dado, pero aboga por una paciente espera por si "el choque llegara a darse en un punto más central" 54 . Como bien puede verse, la vacilación respecto de cuándo se ha llegado a un punto de definición crucial no separaba a amigos y enemigos del pueblo judío, sino que podía separar también a sus amigos. Es precisamente a este estado de vacilación que busca responder la fórmula (sin un mayor éxito, como puede apreciarse).

Se trata entonces no solo de fidelidad, sino de una claridad que debe servir para excluir el error con la prisa debida. En ese sentido, se trata de algo muy distinto de un fino ejercicio de revisión de posiciones confesionales, por vinculante que también ese tipo de ejercicio pueda resultar. Además, aunque el lenguaje parece remitir a la existencia de una confesión fija que debe hacerse oír, en realidad lo que está en juego es la confesión de algo que no está explicitado en los textos confesionales. El status confessionis es, pues, no solo un llamado a considerar el punto del que en un contexto específico todo parece pender. Es también un llamado que pasa por sacar a luz, como algo dotado de plena autoridad, lo que previamente solo era implícitamente confesado. Se trata, cabría además precisar, de explicitar una aplicación ética de lo que era dogmáticamente confesado (en este caso, que el bautismo - y no la raza- es el único criterio de pertenencia a la Iglesia).

52 DBW XII, 361.

53 DBW XII, 360. Las "tesis sobre el parágrafo ario", del mismo año, desarrollan una posición similar. Véase DBW XII, 408-415

54 Véase DBW XII, 124-125 para la carta de Bonhoeffer a Barth, 125-128 para la respuesta. 
Si nos dirigimos ahora a 1940, podemos considerar uno de los casos en que Bonhoeffer usa de modo más específico los documentos confesionales. El caso que nos interesa se encuentra en la correspondencia suscitada tras la difusión de una circular sobre la eucaristía, enviada por Bonhoeffer en febrero de 1940 a pastores de la Iglesia Confesante (muchos dispersos ya en el frente). Eberhard Baumann, oficial de un sínodo calvinista con quien Bonhoeffer había además tenido un fructífero trabajo en común en los comienzos de la Iglesia Confesante ${ }^{55}-$, reaccionó contra la fundamentación de tal reflexión en textos de un documento confesional luterano, el capítulo VII de la Confesión de Ausburgo. La primera carta en el intercambio, de Baumann a su par luterano Onnasch, no es una formulación de una doctrina calvinista en respuesta a una luterana, sino una característica diatriba anticonfesionalista. Baumann, que describe la circular de Bonhoeffer como una bula de excomunión, reprocha el carácter "escolástico" de la misiva y todo termina en una queja contra un "confesionalismo cuya estrechez y autojusticia definitivamente no viene de Dios" $" 56$.

La carta fue remitida a Bonhoeffer, quien en su respuesta a Baumann se manifiesta dudoso sobre si todo se trata de un malentendido, o si acaso su interlocutor le está escribiendo "no desde una perspectiva confesional calvinista [reformiert], sino desde una liberal" ${ }^{57}$. Señala ahí que la posición de los escritos confesionales luteranos estaba simplemente siendo presentada como un texto adecuado para orientar la reflexión de los pastores. La legitimidad de tal uso de las confesiones a Bonhoeffer le parece estar fuera de toda duda, y su carta no retrocede un ápice respecto de la importancia teológica que asigna al trabajar sobre la base de lo establecido "por nuestros padres" $"$. Al mismo tiempo, con todo, Bonhoeffer deja entrever que no se trata de una posición que esté asumiendo íntegramente; de hecho, escribe que "personalmente me aparto de la Fórmula de Concordia en

55 Al respecto véase E. Bethge, Dietrich Bonhoeffer. Theologe, Christ, Zeitgenosse 501 y 538. A pesar de sus reservas ante el luteranismo de Bonhoeffer, Baumann solía enviar a sus mejores estudiantes a pasar un periodo bajo la dirección de éste en el seminario ilegal de Finkenwalde.

56 DBW XVI, 15-16.

57 DBW XVI, 17.

58 DBW XVI, 18. 
varios puntos ${ }^{\prime 59}$. El punto en discusión, sobre la Cena del Señor, es uno en el que Bonhoeffer señala haber modificado su posición precisamente "a causa del exhaustivo trabajo teológico de la Institución de Calvino"60. Es crucial notar el modo preciso en que esta modificación de su juicio personal se plantea, pues si bien muestra disposición a apartarse de la Fórmula de Concordia, tal flexibilidad no está siendo planteada en términos doctrinalmente minimalistas ni tampoco se está planteando en términos de una apelación directa a la Biblia, sino que es un cambio producto del enfrentamiento con otro texto doctrinalmente robusto, como es la Institución.

Bien cabe decir que Baumann y Bonhoeffer llegan tras esto a sustantivo acuerdo. Pues en la siguiente carta de Baumann ya desaparece del todo la retórica anticonfesional, y es reemplazada por una serie de observaciones primordialmente prudenciales sobre el modo en que tal trabajo de reflexión sobre textos del siglo XVI debería realizarse. Hay en dicha carta una legítima preocupación por aquellos a quienes "su reforma se les ha vuelto canon, regula fidei", y que ni siquiera ante la dramática desintegración bajo el nacionalsocialismo son capaces de concebir que estén en necesidad de profunda revisión ${ }^{61}$. Pero habiendo recibido las explicaciones de Bonhoeffer, Baumann ya no postula un simple biblicismo como cura para tal situación, sino el aproximarse a estos asuntos desde el conjunto de los textos confesionales del siglo XVI y con expreso planteamiento de las preguntas que debieran guiar la reflexión contemporánea. Baumann, en otras palabras, consideraba poco realista la visión que Bonhoeffer parecía tener de los lectores de sus circulares teológicas, como si por cuenta propia pudiesen conducir tales reflexiones ${ }^{62}$. Es probable, con todo, que la razón para la ausencia de una guía fuera en realidad muy distinta: no exceso de fe en los alumnos, sino falta de fe en los profesores. Según Bonhoeffer confiesa a Barth en carta, no creía que hubiera en Alemania nadie capaz de conducir adecuadamente la necesaria discusión entre ambas tradiciones

\footnotetext{
59 DBW XVI, 17.

${ }_{60}$ DBW, XVI, 17.

${ }^{61}$ DBW XVI, 27.

62 DBW XVI, 26.
} 
confesionales $^{63}$. En cualquier caso, Bonhoeffer cierra la correspondencia con Baumann en sustantivo acuerdo con el mismo ${ }^{64}$.

Podemos concluir atendiendo al uso que Bonhoeffer preveía para las confesiones en un futuro tras la caída del régimen nacionalsocialista. En 1943 ya se había dado el giro por el que tanto fuera como dentro de Alemania la derrota de esta empezaba a verse como segura. En tal contexto no solo comenzó una intensa reflexión entre intelectuales cristianos de las potencias aliadas, que temían por un futuro en el que triunfaran ante Alemania solo por superioridad técnica y no por riqueza espiritual ${ }^{65}$. También dentro de Alemania los variados círculos de resistencia comenzaron a extender su reflexión desde el presente al futuro ${ }^{66}$. Hasta poco antes de su detención, Bonhoeffer mismo estuvo trabajando en un texto -que nació no del contexto eclesiástico, sino de los círculos de la resistencia vinculada a la inteligencia militar (Abwehr) - titulado "Bosquejo para la reorganización de la Iglesia tras el final del Kirchenkampf". Ahí Bonhoeffer habla respecto de la Iglesia Evangélica Alemana (DEK) como una institución cuya unidad podría ser reestablecida perfectamente una vez reconocida una administración que operara en conformidad con las confesiones ${ }^{67}$. Por una parte, advierte contra una simple vuelta al orden eclesiástico subsistente en la unión prusiana hasta 1933, el orden en que una autoridad central contiene las diferencias confesionales; pero, por otra parte, sugiere que esta alternativa permitiría que lentamente superen sus escrúpulos "algunas iglesias regionales enraizadas en ciertas tradiciones históricas y confesionales" ${ }^{\text {" }}$. Esta formulación puede no ser todo lo clara que desearíamos, pero consiste una vez más en una suerte de vía media. Ella busca reconocer un lugar fundamental a los textos confesionales, pero al mismo tiempo marca distancia respecto de un confesionalismo percibido

${ }^{63}$ DBW XIV, 238.

${ }^{64}$ DBW XVI, 25-27 y 35.

${ }^{65}$ Sobre dichos esfuerzos véase A. JACOBS, The Year of Our Lord 1943. Christian Humanism in an Age of Crisis (Oxford University Press, Oxford, 2018).

${ }^{6}$ Para estos círculos véase H. Mommsen Alternatives to Hitler. German Resistance under the Third Reich (Princeton University Press, Princeton, 2003), en particular el capítulo "The Social Vision and Constitutional Plans of the German Resistance".

${ }^{67}$ DBW XVI, 592-593. Véase también DBW XVI, 590 para el modo en que esto es planteado como continuación del carácter confesional de la iglesia confesante. 
como crudamente tradicionalista. El confesionalismo formalista e incapaz de revisión, que otros textos personifican en Sasse, aquí se identifica con el orden eclesiástico de los estados completos de Württemberg y Baviera. La esperanza de Bonhoeffer inequívocamente era la de ver un cambio en estos grupos, pero evidentemente el cambio esperado no era el de una pérdida de su confesionalidad.

\section{Conclusión}

Bonhoeffer no fue un "fanático de las confesiones" (una crítica que recibían al menos algunos sectores de la Iglesia Confesante) ${ }^{69}$. Pero mientras otros llamaban a dejar de hablar y en lugar de eso actuar, él advertía que "retroceder desde la palabra hacia la acción, desde la confesión hacia el amor, sería cobardía, sería eludir nuestra condición de mensajeros, sería evitar la dificultad de armonizar nuestro hablar y nuestro actuar" ${ }^{\text {"70 }}$. Estas palabras de 1935, de un curso de homilética en el seminario clandestino de Finkenwalde, puede constituir un adecuado punto de referencia para poner en perspectiva la preocupación de Bonhoeffer por las confesiones de fe: la suya es una aproximación para la que la armonía entre el actuar y el hablar es relevante, pero se trata a la vez de una aproximación que no quiere alcanzar dicha armonía por un simple ajuste de nuestro hablar al modo en que actuamos. Entronca, por el contrario, con un conjunto de tradiciones protestantes que no solo mantienen la centralidad de la palabra revelada en un principio, sino que también confieren valor normativo, por subordinado que fuere, a las confesiones surgidas en momentos críticos de la historia de la Iglesia. Las referencias generales de Bonhoeffer y otros miembros de la Iglesia Confesante a la importancia de "defender la confesión" pueden en principio parecer vagas, y no siempre está claro qué elementos del legado de la Reforma son los que se procura seguir confesando. Nuestra revisión ha mostrado, con todo, que una lectura atenta revela preocupación tanto por el efecto que tiene la confesión de un entramado completo de creencias como por la capacidad de sacar de dicho entramado conclusiones hasta entonces implícitas.

69 Así, por ejemplo, en carta de Helmut Rössler a Bonhoeffer 6.12.1934. DBW XIII, 254.

70 DBW XIV, 479. mi cursiva. 
La "confesión de fe" no ha, pues, de ser reemplazada por una "confesión vital", pero tampoco por la sola apelación a las Escrituras. Hemos visto que esta posición, que lo aleja tanto de los germano-cristianos como de posiciones biblicistas, moldea el actuar de Bonhoeffer en distintos momentos y ante las más variadas tareas: a la hora de identificar la necesidad más urgente del presente, a la hora de buscar la conciliación entre los hermanos divididos, a la hora de pensar el futuro de postguerra de su país. En cada uno de esos pasos hemos visto su intento por dar con alguna vía media que permitiera seguir describiendo como vinculante su adhesión a las confesionales, al mismo tiempo distanciándolo del confesionalismo infructífero por el que caracteriza tanto a algunos teólogos como a algunos de los estados alemanes. Si acaso en cada uno de estos pasos Bonhoeffer logra el equilibrio deseado es, desde luego, una pregunta distinta. Pero lo decisivo para comprender su singular posición es el ahínco con que se aferra a que haya modos de sustantiva adhesión a las confesiones que tengan también en el presente un papel a desempeñar.

Ese tipo de preocupación en buena medida puede ser nuestra también. Bonhoeffer nos ayuda a preguntar por la necesidad y características de un confesionalismo apropiado para una época post-confesional. En ella con frecuencia se asume que la humildad epistemológica necesaria para la paz debiera tener por efecto una menor intensidad en la confesión y una menor extensión en la cantidad de cosas confesadas. La Iglesia Confesante y el caso particular de Bonhoeffer parecen importantes contraejemplos para esa narrativa. La ausencia de un entramado doctrinal puede en realidad volver importante cualquier cosa indiferente y así desatar el fanatismo (no en vano Bonhoeffer caracteriza el fanatismo no por la intensidad de sus convicciones, sino por perder de vista el todo del mal, ocuparse de lo no esencial, y así, cual toro, dar en el paño rojo en lugar de al torero) ${ }^{71}$. Por lo demás, los mismos estudiantes de Bonhoeffer se planteaban a propósito de la Confesión de Betel un propósito de indiscutible vigencia. Buscaban que mediante esta también la contraparte se viera confesada a explicitar. Dicha expectativa cobra peculiar importancia en un contexto pluralista. Si vamos a tener un tipo de pluralismo que permita la efectiva manifestación de las distintas visiones de mundo, con miras a su real conocimiento y al intercambio esclarecedor, el hábito de explicitar las

${ }^{71}$ DBW VI, 64-65; de modo casi idéntico en DBW VIII, 21-22. 
propias creencias debe entrenarse. Ya una confesión tempranomoderna como el Consensus Tigurinus, de 1549, había planteado esto como uno de los efectos que tendría que tener una confesión. En sus palabras, "los que parecen disentir, o de hecho disienten, deben expresar su visión de modo abierto y por turnos, tanto de modo oral y por un documento escrito, realizando una declaración mediante formulaciones definidas, apropiadas y significativas" ${ }^{72}$. Se trata de un espíritu explícitamente recogido por Bonhoeffer en medio de la discusión en torno a la Confesión de Betel. Ante el clima de escepticismo por lo que lograría la redacción de dicha confesión, Bonhoeffer insiste en que ella al menos crea las condiciones para una respuesta. "Queremos que digan lo que quieren. Si acaso lo lograremos, es otra cosa", escribe ${ }^{73}$. "La pregunta final -insiste- será simplemente germanismo o cristianismo, y cuánto antes el conflicto salga a la luz, tanto mejor" ${ }^{374}$. No es ésa la disyuntiva que enfrenta nuestra sociedad, y nuestra meta puede ser distinta del mero sacar a la luz un conflicto. Pero Bonhoeffer no parece equivocarse respecto del papel que pueden desempeñar los documentos confesionales para orientar y explicitar disyuntivas.

Citado por Pelikan, Credo 252.

73 DBW XIII, 117-118.

74 DBW XIII, 118. 